\title{
Les écoles de médecine en Afrique subsaharienne ${ }^{\star}$
}

\section{Medical schools in sub-Saharan Africa}

Fitzhugh Mullan ${ }^{1}$, Seble Frehywot, Francis OMaswa ${ }^{18}$, Eric BuCH ${ }^{2}$, Candice CHEN, S Ryan GrEYSEN ${ }^{3}$, Travis WAssermanN ${ }^{1}$, Diaa Eldin ElGaili ABUBAKR ${ }^{4}$, Magda AwASEs ${ }^{19}$, Charles BoeleN ${ }^{17}$, Mohenou Jean-Marie Isidore Diomande $^{1}$, Delanyo Dovlo ${ }^{6}$, Josefo Ferro ${ }^{7}$, Abraham HaIleAmlaK ${ }^{8}$, Jehu IPUto ${ }^{9}$, Marian Jacobs ${ }^{10}$, Abdel Karim Koumaré $^{11}$, Mwapatsa MiPAndo ${ }^{12}$, Gottleib Lobe MoneKosso ${ }^{13}$, Emiola Oluwabunmi OlaPADE-OlaOPA ${ }^{14}$, Paschalis Rugarabamu $^{15}$, Nelson K SEwANKAMbo ${ }^{16}$, Heather Ross ${ }^{1}$, Huda AYas ${ }^{1}$, Selam Bedada CHALE ${ }^{1}$, Soeurette CYPRIEN ${ }^{1}$, Jordan CoheN $^{1}$, Tenagne HAILE-MARIAM ${ }^{1}$, Ellen HAMBURGER ${ }^{1}$, Laura JolleY ${ }^{1}$, Joseph C Kolars ${ }^{20}$, Gilbert KombE ${ }^{21}$ et Andre-Jacques NEUSY ${ }^{22}$

1 The George Washington University, Washington, DC, USA

2 University of Pretoria, Pretoria, Afrique du Sud

3 Yale University, New Haven, CT, USA

4 University of Gezira, Wad Madani, Soudan

5 University of Cocody, Abidjan, Côte d'Ivoire

6 World Health Organization, Geneva, Suisse

7 Catholic University of Mozambique, Beira, Mozambique

8 Jimma University, Jimma, Ethiopie

9 Walter Sisulu University, Mthatha, Afrique du Sud

10 University of Cape Town, Cape Town, Afrique du Sud

11 University of Mali, Bamako, Mali

12 University of Malawi, Blantyre, Malawi

13 Global Health Dialogue Foundation, Yaounde, Cameroun

14 University of Ibadan, Ibadan, Nigeria

15 Hubert Kairuki Memorial University, Dar Es Salaam, Tanzanie

16 Makerere University, Kampala, Ouganda

17 International Consultant in Health Systems and Personnel, Sciez-sur-Léman, France

18 African Center for Global Health and Social Transformation, Kampala, Ouganda

19 World Health Organization, Harare, Zimbabwe

20 University of Michigan Medical School, Ann Arbor, MI, USA

21 Abt Associates, Bethesda, MD, USA

22 New York University School of Medicine, New York, NY, USA

Mots clés : Afrique subsaharienne ; facultés de médecine ; santé publique
Résumé - Contexte, problématique et objectifs : Les nombres restreints de diplômés sortant d'écoles de médecine peu nombreuses, et l'émigration des diplômés vers d'autres pays contribuent au faible nombre de médecins présents en Afrique subsaharienne. L'étude

^ La version princeps de cet article a été publiée en anglais dans The Lancet (Mullan F, Frehywot S, Omaswa F, Buch E, Chen C, Greysen SR et al. Medical Schools in Sub-Saharan Africa. Lancet 2011;337:1113-21. Copyright Elsevier, 2011).

La présente traduction française a été effectuée par Odile Bosch 4602 Cornwallis Court 22309 Alexandria (VA), USA, et révisée au sein du comité de rédaction de Pédagogie Médicale ; bien qu'elle ait été effectuée avec la permission des éditions Elsevier, elle n'a pas révisée sous l'égide de cet éditeur. 


sur les écoles de médecine en Afrique subsaharienne a examiné les défis, les innovations et
les nouvelles tendances au regard de l'éducation en médecine dans la région. Méthodes :
Nous avons retenu 168 écoles de médecine ; parmi les 146 qui ont été soumises à l'enquête,
$105(72 \%)$ ont répondu. Résultats : Les observations tirées de l'étude indiquaient que les
pays mettaient en priorité l'éducation médicale dans le cadre du renforcement du secteur de
la santé et nous avons constaté nombre d'innovations dans la préparation " pré-médicale»,
l'éducation en équipe et l'usage créatif du soutien limité en matière de recherche. L'étude
a aussi attiré l'attention sur le problème généralisé d'un effectif insuffisant de professeurs
capables d'enseigner les sciences fondamentales et cliniques, d'une infrastructure physique
peu développée et du manque d'accréditation externe. Les tendances enregistrées incluent la
croissance des écoles de médecine privées, l'éducation axée sur la communauté, les partena-
riats internationaux et l'effet positif de la recherche pour le développement des professeurs.
Dix recommandations orientent l'initiative de renforcement de l'éducation en médecine en
Afrique subsaharienne.
Abstract - Context, problematic and objectives: Small numbers of graduates from few
Keywords:
mub-saharian Africa;
medical schools;
public health

\section{Introduction}

La santé en Afrique est importante au regard de la question de l'équité entre les hommes et est un déterminant de la réduction de la pauvreté et du développement humain. L'Afrique supporte $24 \%$ du fardeau total mondial des maladies mais ne compte que $3 \%$ de l'effectif mondial en matière de santé ${ }^{[1]}$. La Joint Learning Initiative ${ }^{[2]}$ (initiative conjointe de recherche) et le Rapport mondial de la santé $2006^{[1]}$ ont révélé la pénurie particulièrement grave des ressources humaines au sein du secteur de la santé en Afrique. Les premières réactions à la prise de conscience de ces manques ont comporté des demandes d'accroissement du nombre des professionnels de santé ${ }^{[3]}$ et de cliniciens non médecins ${ }^{[4]}$, ainsi qu'une réorientation des tâches pour une utilisation plus efficace des cadres de professionnels existants ${ }^{[5]}$. L'attention porte désormais sur l'éducation et la fidélisation des docteurs en médecine en Afrique, non pas parce que les médecins résoudront les immenses besoins non satisfaits du continent en matière de santé mais par conviction qu'aucun système de santé ne peut fonctionner sans un corps adéquat de médecins qui peuvent jouer le rôle de cliniciens, de professionnels de santé du secteur public, de gérants, d'éducateurs et de décideurs ${ }^{[6]}$. L'Afrique subsaharienne compte environ 145000 médecins $^{[7]}$ (5\% des 2877000 médecins en exercice en Europe) pour desservir une population de 821 millions (plus que la population de l'Europe) ${ }^{[8]}$. Globalement, en Afrique subsaharienne la proportion de médecins par rapport à la population est de 18 pour 100000 , chiffre 
à comparer à ceux de pays comme l'Inde (60 pour $100000)$, le Brésil (170 pour 100000$)$ et la France (370 pour 100000$)^{[8]}$. Les pays d'Afrique les plus pauvres font face à des pénuries encore plus grandes à cet égard.

Les taux très faibles de médecins par rapport à la population dans les pays d'Afrique subsaharienne sont dus à nombre de facteurs, parmi lesquels un faible nombre d'écoles de médecine (ce terme étant employé au sens générique pour désigner autant les écoles de médecine que les composantes médicales des universités) d'où sort un nombre modeste de diplômés, l'émigration de nombreux diplômés vers d'autres pays ou continents. Tout effort visant à améliorer la performance du système de santé dans ces pays doit envisager des options s'attachant à accroître la productivité des écoles de médecine et à mieux fidéliser les diplômés dans leurs pays.

L'investissement stratégique dans l'éducation médicale en Afrique subsaharienne suscite un intérêt croissant à l'échelle nationale et internationale mais il existe peu de données sur le statut des écoles de médecine ou sur les tendances au sein de l'éducation médicale dans l'ensemble du continent. Par exemple, lorsque nous avons initialement passé en revue toutes les bases de données possibles relatives aux écoles de médecine en 2008 (OMS, Institute for International Medical Education (Institut pour l'éducation médicale internationale), Foundation for Advancement of International Medical Education and Research (Fondation pour le développement de l'éducation et de la recherche médicale internationales) et World Federation for Medical Education (Fédération mondiale pour l'éducation médicale)), nous avons répertorié 103 écoles; pourtant, notre travail d'enquête a révélé 168 écoles fonctionnant en Afrique subsaharienne. Ce manque de données et de perspective panafricaine pose un gros défi pour les gouvernements africains et les organisations de bailleurs de fonds, qui cherchent à s'atteler à la pénurie de médecins.

L'étude sur les écoles de médecine en Afrique subsaharienne a tenu compte de cette lacune en mettant au point une base d'informations sur le statut, les tendances et les perspectives au regard de l'éducation en médecine africaine pour les éducateurs, les décideurs et les organisations internationales. La figure 1 souligne la structure, les participants et la séquence des activités qui composent cette étude.

\section{Constats}

\section{Résultats généraux}

Parmi les 168 écoles de médecine, 146 ont été contactées avant la clôture de la période d'enquête de l'étude en décembre 2009; 105 (72 \%) écoles ont répondu à cette dernière. (Toutes les écoles contactées sont indiquées dans le matériel éditorial complémentaire en ligne pp. 7-10.) Les pays de population nombreuse $(p<0,0001)$ et de grande superficie $(p<0,0001)$ comptaient plus d'écoles de médecine que les pays plus petits par régression linéaire multiple, mais il n'y avait pas d'indication de nette corrélation entre le produit intérieur brut (PIB) d'un pays par habitant, la région d'Afrique ou la langue nationale et le nombre d'écoles de médecine qui s'y trouvaient. Les répondants à l'enquête et les établissements visités sur place représentaient toutes les régions d'Afrique subsaharienne et tous les principaux groupes linguistiques. Les données collectées lors des visites des sites et de l'enquête sont résumés en 13 catégories.

\section{L'expansion de l'éducation médicale}

Plusieurs pays développent le champ de l'éducation médicale dans le cadre du renforcement du secteur de la santé. Plusieurs gouvernements nationaux investissent énormément dans les ressources humaines pour la santé, produisant des plans stratégiques pour le domaine de la santé, qui incluent une augmentation de l'effectif des professionnels de santé. L'éducation médicale est essentielle au développement 


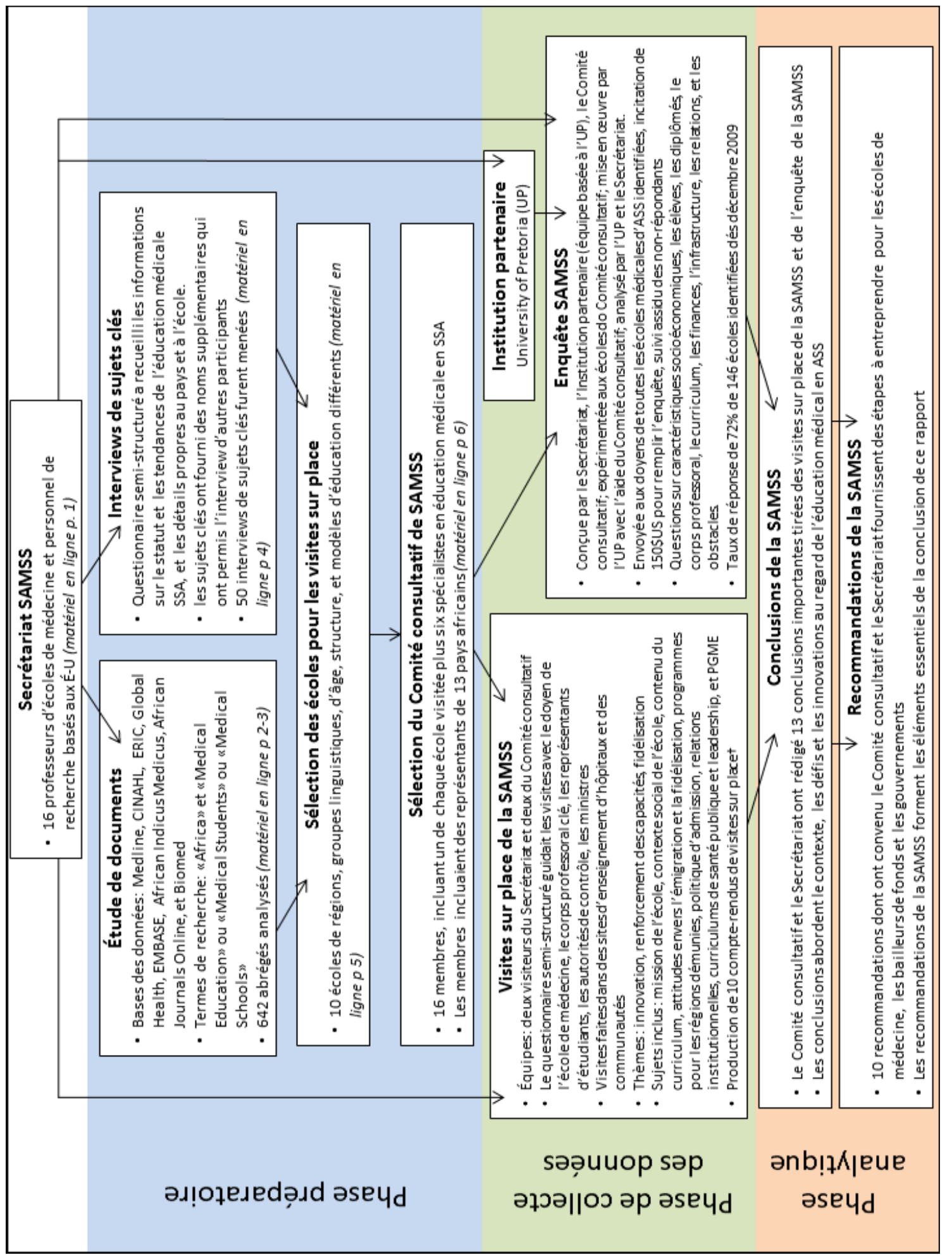

Fig. 1. Structure et méthodes de l'étude sur les écoles de médecine en Afrique subsaharienne. 


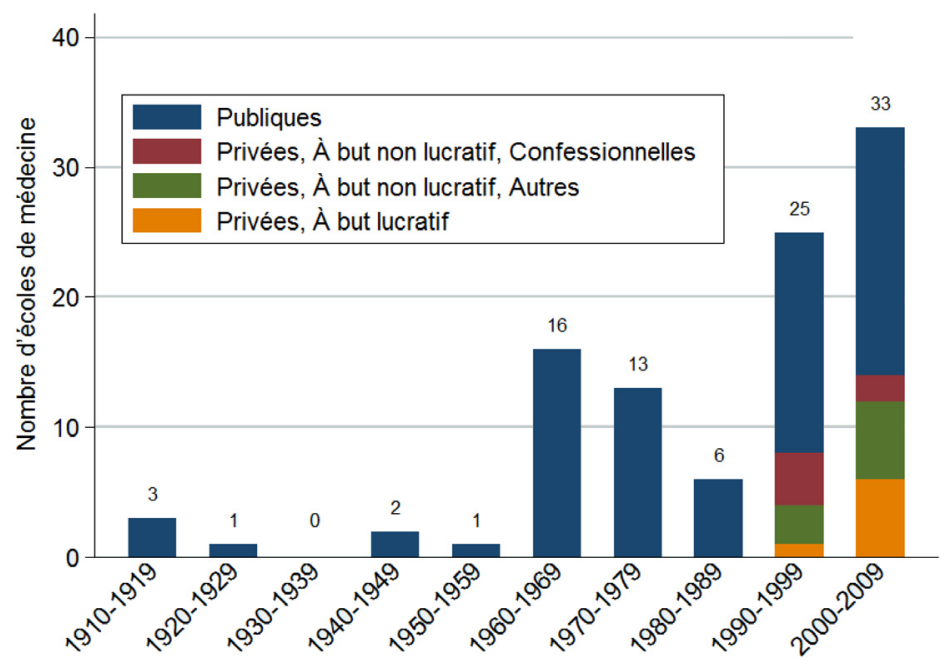

Fig. 2. Dates de fondation des écoles de médecine en Afrique subsaharienne (cinq écoles n'ont pas répondu à l'enquête).

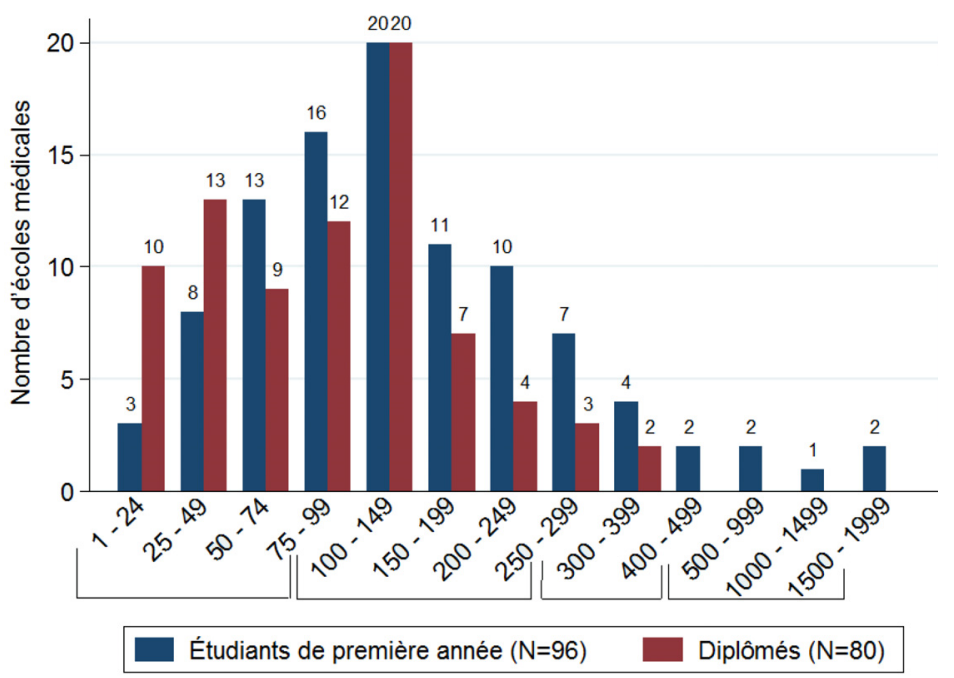

Fig. 3. Nombre d'étudiants en première année et nombre de diplômés dans les écoles de médecine en Afrique subsaharienne (2008). $N$ varie parce que certaines écoles de médecine n'ont pas encore de médecins diplômés.

d'un effectif de professionnels de santé et s'inscrit intégralement dans les plans de ressources humaines. Sept écoles de médecine qui ont répondu ont été fondées avant 1960 et 29 autres lors des décennies d'indépendance (1960-79). Le nombre a peu augmenté pendant les années 80 mais 58 des écoles ayant répondu ont ouvert leurs portes depuis 1990 (figure 2).

Beaucoup d'écoles de médecine augmentent le nombre d'inscriptions d'étudiants. Cinquante-neuf sur $78(76 \%)$ qui ont répondu ont fait état d'un accroissement du nombre d'étudiants de première année, par rapport à il y a cinq ans. Cinquante-six sur $105(53 \%)$ ont fait part de projets d'accroissement au cours des cinq prochaines années, 57 sur 96 (59\%) ayant reçu l'ordre d'augmenter leurs inscriptions par les ministères de la santé ou de l'éducation.

Le nombre total actuel d'étudiants inscrits en première année dans 96 des écoles répondant à l'enquête est 18349 . Le nombre de diplômés dans les écoles qui ont répondu était de 7861 en 2008 (figure 3). Ce nombre représente les diplômés 


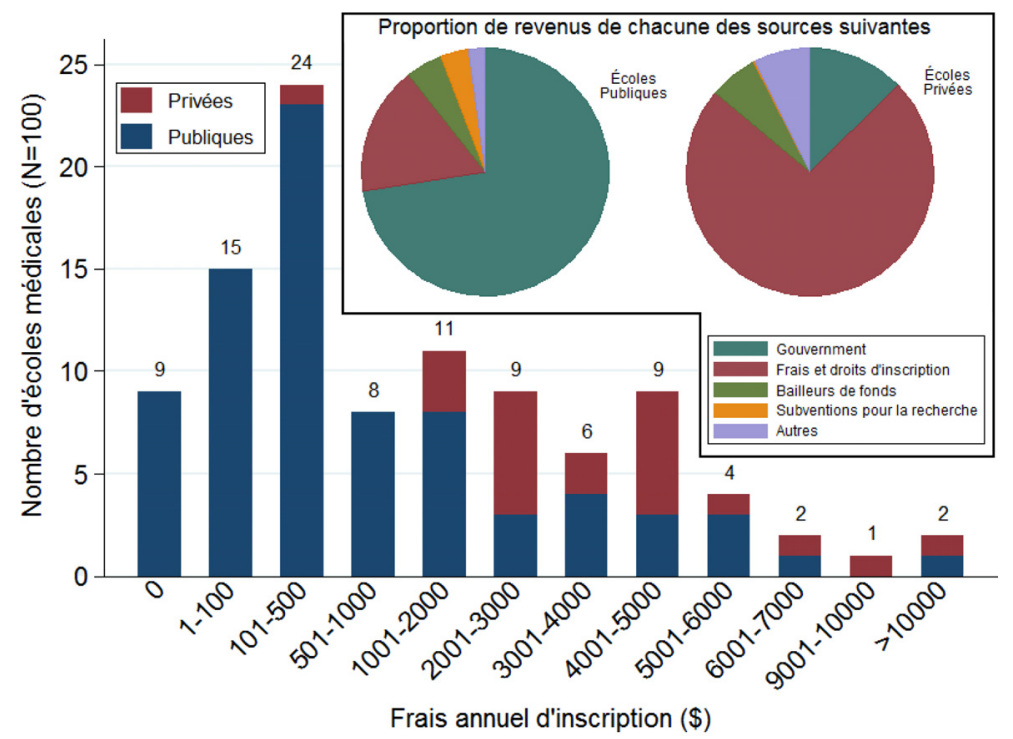

Fig. 4. Frais d'inscription et sources de revenus dans les écoles de médecine en Afrique subsaharienne.

sortant des 105 écoles de médecine qui ont répondu à l'enquête. Nombre des écoles qui n'ont pas répondu à l'enquête sont privées ou récentes et leurs caractéristiques semblent indiquer que le nombre moyen de diplômés est moins grand pour les 63 écoles n'ayant pas répondu que pour les écoles qui ont répondu. Ces données signifient qu'environ 10000 à 11000 diplômés par an sortent des écoles de médecine en Afrique subsaharienne. Les différences entre le nombre d'inscriptions et celui des diplômés sont surtout attribuables à l'ouverture ou à l'expansion d'écoles. Quelques universités acceptent initialement un grand nombre d'étudiants et réduisent cet effectif en deuxième année. Cinquanteneuf écoles sur 84 (70 \%) répondant à l'enquête ont déclaré qu'au moins $80 \%$ des étudiants inscrits en première année sortaient diplômés.

Le gouvernement éthiopien investit largement dans le plan d'amplification de l'effectif suivant la stratégie appelée «flood and retain» (forte augmentation et fidélisation), ce qui implique une augmentation considérable et rapide du nombre de professionnels de santé formés et la prise de mesures de fidélisation correspondantes. Le ministère de l'éducation a ordonné que toutes les écoles de médecine augmentent la taille de leurs promotions. Ainsi, les inscriptions en première année à Jimma University (Éthiopie) sont passées de 200 à 250 en 2009 et devraient atteindre 350 en 2011. Le gouvernement appuie cette stratégie en investissant dans l'infrastructure matérielle, incluant la construction d'un nouvel hôpital universitaire. L'Hubert Kairuki Memorial University en Tanzanie illustre l'augmentation du secteur privé, en augmentant ses admissions de 25 étudiants en première année de médecine, au départ en 1998, à 70 par an en 2010. Le gouvernement tanzanien a apporté son soutien en octroyant des prêts et des bourses aux étudiants des écoles privées, donnant les moyens à davantage d'étudiants de payer les droits d'inscription.

Dans toutes les écoles de médecine en Afrique subsaharienne, les tarifs varient beaucoup. Neuf écoles $(9 \%)$ répondant à l'enquête offrent une inscription gratuite, 47 (47\%) font payer 1000 \$ US ou moins par an et neuf $(9 \%)$ demandent plus de 5000 \$. La plus grande partie des revenus des écoles privées proviennent des frais d'inscription; les écoles publiques reçoivent la plupart de leurs budgets d'exploitation du gouvernement (figure 4).

En réponse à une question ouverte, les répondants devaient discerner les trois besoins majeurs qui justifiaient une augmentation de la qualité et 


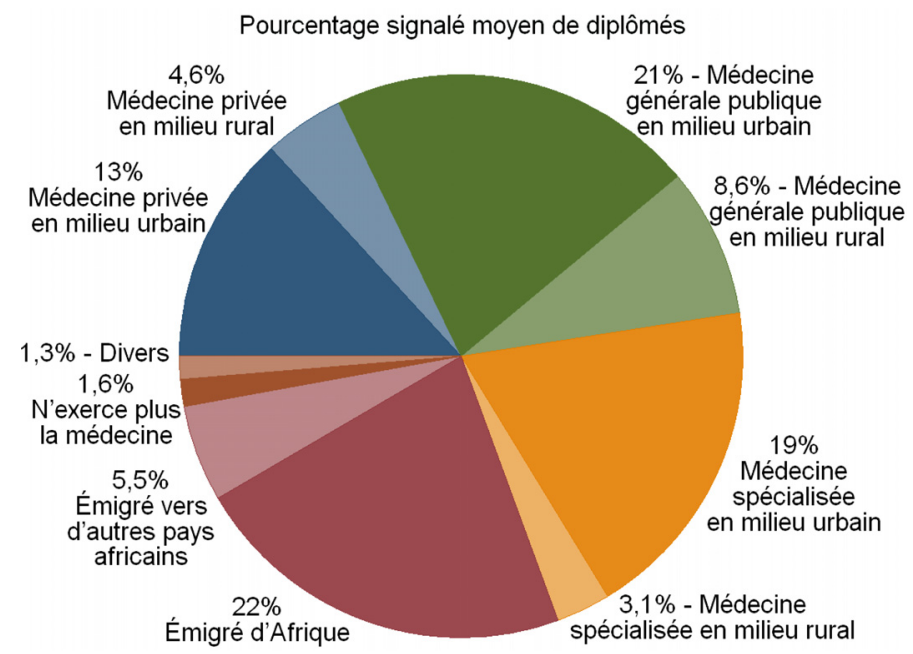

Fig. 5. Localisation (estimation) des diplômés d'écoles de médecine d'Afrique subsaharienne cinq ans après leur diplôme (données exprimées en pourcentage sgnalé moyen de diplômés $(N=62)$.

du nombre de leurs diplômés. La page 11 du matériel éditorial complémentaire en ligne récapitule les réponses. Les aspects se rapportant au corps professoral étaient le plus souvent identifiés comme essentiels pour améliorer la qualité des diplômés (35 répondants sur 94 ont qualifié ce point comme étant le plus important). Les questions d'infrastructure étaient le plus souvent considérées comme importantes pour améliorer le nombre de diplômés (37 répondants sur 94 ont noté cette question comme la plus importante). Les éléments du curriculum étaient perçus comme ayant un impact sur la qualité, alors que les améliorations des sites cliniques pouvaient améliorer la quantité. Les réponses faisaient allusion aux problèmes budgétaires en lien avec les deux questions.

\section{Effet du système de santé du pays}

Lorsque la société civile est désorganisée et que l'efficacité de la gouvernance est réduite, l'éducation médicale et la fidélisation des médecins s'en trouvent affectées. Les diplômés de nombreux pays refusent de travailler dans des régions rurales à cause du manque d'appui clinique. Les diplômés de l'Ibadan University, par exemple, renoncent aux emplois du vaste et crucial réseau d'hôpitaux secondaires du Nigeria à cause des salaires et conditions de travail médiocres, ainsi que des pénuries de fournitures, de personnel de soutien et d'équipement.

L'évaluation des stratégies de fidélisation a posé des défis parce que la plupart des systèmes de santé ont peu de moyens permettant suivre les diplômés des écoles de médecine. Quarante-sept sur 58 (81\%) répondants à l'enquête dont les écoles ont produit des médecins diplômés ont dit qu'ils n'avaient pas de système de suivi mis en place. La figure 5 indique les endroits où se trouvent les diplômés des écoles de médecine cinq ans après l'obtention de leur diplôme selon l'estimation de 62 écoles répondant à cette question de l'enquête. Le pourcentage estimé de diplômés exerçant la médecine générale en milieu rural cinq ans après la fin de leurs études était incontestablement corrélé à l'existence d'un programme de service obligatoire $(p=0,039)$, au faible nombre de programmes de troisième cycle d'études médicales $(p=0,016)$, et au fait que l'enseignement soit dispensé en français ( $p=0,016)$; ces données ont été produites par analyse de co-variance, ANCOVA. Nous n'avons pas remarqué de corrélation importante avec le PNB, l'existence d'un programme de recrutement ciblé pour les étudiants de milieu rural, le pourcentage de la population nationale en milieu 
Tableau I. Groupe de travail : recommandations pour avancer et améliorer l'éducation médicale et la santé de la population en Afrique subsaharienne.

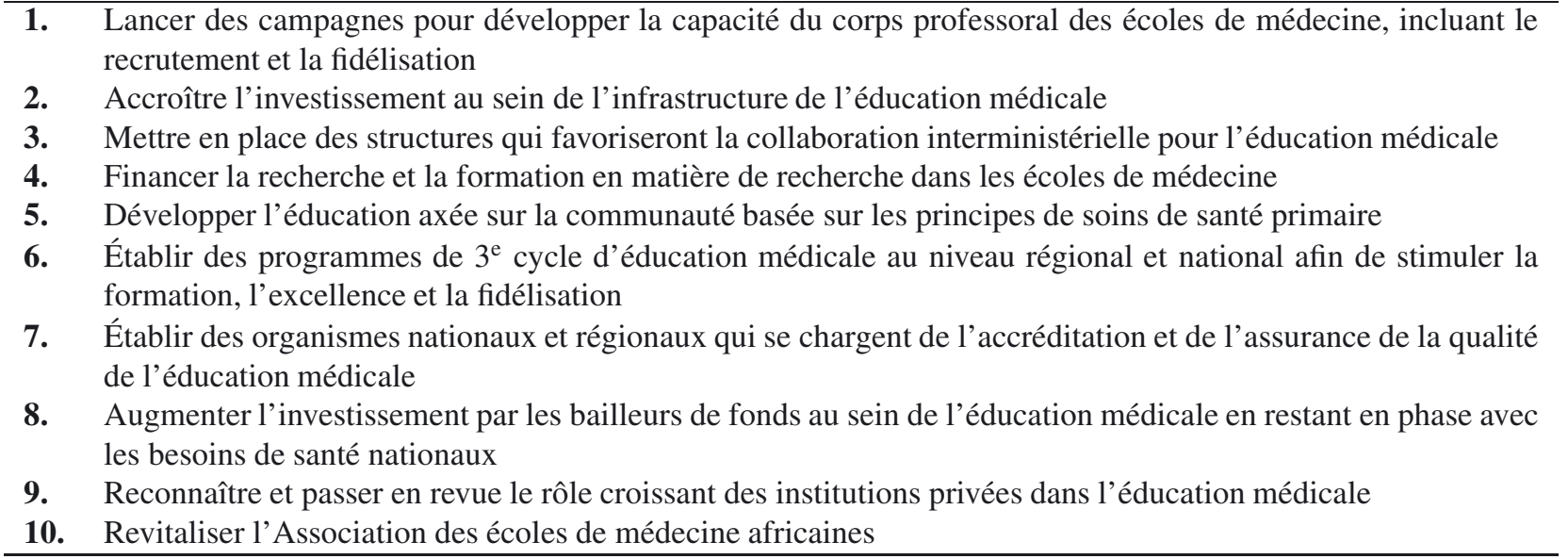

rural ou l'orientation du dispositif d'éducation sur la communauté. Nombre d'écoles et de pays tentent de faire face à l'émigration. Le service national est requis pour les diplômés du Mozambique, d'Afrique du Sud, d'Éthiopie et du Nigeria afin d'obtenir un service clinique de la part de tous les diplômés, bien que la mise en vigueur de ces exigences soit variable.

\section{Pénuries au sein des installations des écoles de médecine}

Les pénuries au sein des corps professoraux des écoles de médecine sont endémiques, problématiques et aggravées par l'émigration des professionnels de santé. Pratiquement toutes les écoles que nous avons visitées dans le cadre de cette enquête indiquaient un certain manque de professeurs au regard des sciences fondamentales et cliniques. L'effectif du personnel enseignant (salarié à temps complet ou partiel et bénévole) dans 51 parmi 98 écoles répondant à l'enquête est inférieur à 100 ; environ la moitié ont entre $52\left(25^{\mathrm{e}}\right.$ percentile) et $147\left(75^{\mathrm{e}}\right.$ percentile) professeurs. Les salaires modestes, les options limitées de carrière, les charges de cours importantes, les inscriptions croissantes et l'absence d'équipement et de personnel de soutien sont les principaux obstacles à la fidélisation du corps professoral dans les universités.
Les pénuries aggravent encore les contraintes pour le corps professoral et favorisent l'émigration ou le changement d'affectation dans des organisations privées ou des recherches de postes au sein d'organisations non gouvernementales. Les membres du corps professoral bien formés et accrédités sont d'excellents candidats pour le recrutement à l'extérieur du pays, ce qui appauvrit à la fois le corps des cliniciens et celui des professeurs.

Les salaires académiques restreignent sérieusement le recrutement et la fidélisation du corps professoral. Dans beaucoup d'universités, le personnel clinique est payé suivant le même barème de traitement que d'autres professeurs d'université, qui est inférieur à celui des médecins du secteur public fixé par les ministères de la santé. Les opportunités de recherche sont souvent rares et les responsabilités de professeurs sont lourdes. À la Gezira University au Soudan, l'absence de spécialistes en sciences fondamentales implique que les cliniciens doivent souvent enseigner les sciences fondamentales aux étudiants en médecine. Beaucoup d'écoles dépendent de professeurs expatriés. Les membres du corps professoral fondateurs de la Walter Sisulu University en Afrique du Sud étaient originaires d'Ouganda, de Cuba et du Nigeria. Certaines écoles ont mis en place des stratégies créatives afin de fidéliser leurs professeurs, telle que la Hubert Kairuki Memorial 
University en Tanzanie, qui offre des incitations comme le logement gratuit, des indemnités pour les communications, du temps d'antenne gratuit et une participation à des séminaires.

La Catholic University au Mozambique a ciblé ses efforts afin de former et d'aider le corps professoral de l'école de médecine. Aujourd'hui, plus de la moitié de leur corps professoral comprend des Mozambicains, bien que l'université reste encore tributaire d'expatriés. À la Walter Sisulu University, le manque de personnel clinicien est atténué en grande partie par des partenariats avec des cliniciens des hôpitaux locaux, qui sont employés par le Département provincial de santé mais dont les contrats les obligent à participer à l'enseignement.

La perte de professeurs au sein des écoles sollicitées dans le cadre de l'enquête était substantielle, avec en moyenne une perte de $10 \%$ du personnel en cinq ans, la moitié des écoles perdant entre $6 \%\left(25^{\mathrm{e}}\right.$ percentile $)$ et $18 \%\left(75^{\mathrm{e}}\right.$ percentile $)$ de leur corps professoral en cinq ans. La principale raison donnée pour la perte de professeurs était l'émigration (matériel éditorial complémentaire en ligne p. 12). Au terme de l'analyse par régression linéaire multiple, le pourcentage de postes de professeurs vacants s'avère être le plus bas dans les pays doté d'un PIB par habitant élevé ( $p=0,0084)$ et le plus élevé dans les écoles de médecine publiques ( $p=0,0099)$, par régression linéaire multiple. La plupart des répondants (80 parmi 100) pensaient que la fidélisation des médecins dans leur pays posait des difficultés; cependant, seuls 51 citaient des démarches à prendre au niveau de l'université pour tenter de résoudre le problème; les plus courantes étaient des augmentations de salaires ou de primes (20 répondants), le renforcement des programmes pour l'éducation du troisième cycle (13) et la mise en œuvre d'une éducation axée sur la communauté (9).

\section{Les déficits d'infrastructure en éducation médicale}

Les insuffisances concernant les infrastructures nécessaires à l'éducation médicale sont omniprésentes et restrictives. À la Jimma University, l'électricité, l'eau et les télécommunications ne sont pas fiables, nuisant à la formation et à l'innovation. À l'Ibadan University, les répondants ont fait part de leur inquiétude quant aux pannes d'électricité quotidiennes. Les départements doivent acheter des générateurs pour assurer les fonctions cliniques et d'enseignement. À la Catholic University, les défis incluent un nombre insuffisants d'ordinateurs, des limitations de la connectivité à Internet et l'absence de foyers d'étudiants. Les logements inadéquats à proximité des sites cliniques posent aussi des problèmes à la Walter Sisulu University et à l'Université du Mali.

L'expérience du College of Medicine au Malawi est un bon exemple du rôle des partenariats dans l'amélioration de l'infrastructure. Grâce à des fonds provenant de Suède, de Norvège et du Fonds mondial de lutte contre le Sida, la tuberculose et la malaria, l'école a construit et amélioré des salles de conférence, des bibliothèques, des foyers, des installations informatiques, des bureaux et des zones de loisirs. Ces améliorations permettent de mieux faire face à des effectifs de classes plus nombreux, ainsi qu'à un corps professoral croissant.

L'enquête de l'étude a examiné la qualité et la quantité de ressources matérielles et de communications précises. Par analyse en régression linéaire multiple ont été explorées les relations entre respectivement six scores rendant compte de diverses ressources (les bâtiments, les bibliothèques, les laboratoires, les sites cliniques, Internet et la technologie d'information et de communication moderne [TIC]) et divers facteurs nationaux et institutionnels (matériel éditorial complémentaire en ligne pp. 13-14). Un PIB élevé était associé à des scores élevés pour cinq des six ressources, les écoles plus anciennes ayant obtenu un score élevé pour quatre des ressources et les écoles publiques ayant évalué leurs ressources comme étant les pires pour trois des indicateurs. Les écoles dont les frais d'inscriptions sont les plus élevés ont fait état de ressources en TIC plus modernes que celles dont les frais sont moins importants. 


\section{Coordination entre les ministères de l'éducation et de la santé}

Une coordination insuffisante entre les ministères de l'éducation et de la santé peut empêcher les écoles de médecine d'augmenter la capacité de l'effectif du secteur de la santé. Ce problème était courant dans presque tous les pays que nous avons visités. Le ministère de l'éducation procure généralement les fonds pour les écoles médicales, alors que le ministère de la santé est le principal employeur des diplômés sortant des écoles de médecine. Dans nombre de pays, la planification coordonnée en matière de budgets, priorités et résultats entre les ministères de la santé et de l'éducation est mauvaise, ce qui contribue à des curriculum inappropriés et la remise de diplômes à des médecins qui ne peuvent pas trouver d'emploi dans le pays. Au Mali et au Soudan, le nombre annuel de diplômés dépasse largement la capacité du pays à embaucher de nouveaux médecins, malgré le besoin de services de santé.

Dans l'ensemble, les ministères de l'éducation semblent plus efficaces à établir des priorités pour les écoles de médecine que les ministères de la santé. Parmi les répondants à l'enquête, 69 sur 100 ont dit que les ministères de l'éducation contribuaient largement aux priorités ou en étaient les éléments moteurs majeurs alors que comparativement 49 sur 101 déclarent que les ministères de la santé étaient des contributeurs sérieux ou principaux. Un répondant à l'enquête a mentionné qu'une innovation notable était le transfert de la supervision de l'école du ministère de l'éducation au ministère de la santé.

\section{Accréditation et évaluation de la qualité}

L'accréditation et l'évaluation de la qualité sont des développements importants pour normaliser l'éducation médicale et les capacités des médecins. Divers niveaux d'accréditation et de certification ont été remarqués dans les pays que nous avons visités. En Éthiopie, il n'existe pas d'organisme d'accréditation continue pour les écoles de médecine.
L'accréditation n'est accordée que lors de la fondation d'une institution. Pourtant, nombre d'écoles font état de progrès en matière d'accréditation des établissements et de l'évaluation des diplômés. Au Mozambique, la formation récente du Conseil médical prévoit de mettre au point des normes d'accréditation pour les écoles de médecine et des épreuves d'évaluation externes pour les étudiants en médecine. La Commission tanzanienne pour les universités visite les institutions une fois avant leur accréditation, puis tous les quatre ans. Le Conseil médical du Malawi se sert de directives de la Communauté de développement sud-africaine pour l'assurance de l'accréditation et de la qualité.

\section{Planification éducationnelle axée sur les besoins nationaux en matière de santé}

La planification de l'éducation axée sur les besoins nationaux en matière de santé améliore la capacité des diplômés en médecine à satisfaire ces besoins. Les écoles mettent l'accent sur des programmes d'éducation médicale orientés sur la communauté, pertinents ou privilégiant les priorités nationales.

De nombreuses écoles de médecine mettent au point des curriculums portant sur les problèmes de santé de priorité nationale et se servent d'expériences en milieu rural et axées sur la communauté pour améliorer leurs programmes. Bien que certaines initiatives soient lancées par les écoles seules, beaucoup d'entre elles s'inscrivent dans le contexte de priorités du gouvernement et des programmes de service national. Au Malawi, le curriculum est conçu pour immerger les étudiants dans les questions de santé locales. Le curriculum se concentre sur les maladies et les troubles de santé les plus courants au Malawi et dans la région. À la Gezira University, les cours axés sur la communauté représentent $25 \%$ des études et nombre de cours ont lieu sur le terrain, y compris dans les hôpitaux de districts, les centres de santé communautaires, les cliniques et chez les patients. La Catholic University 


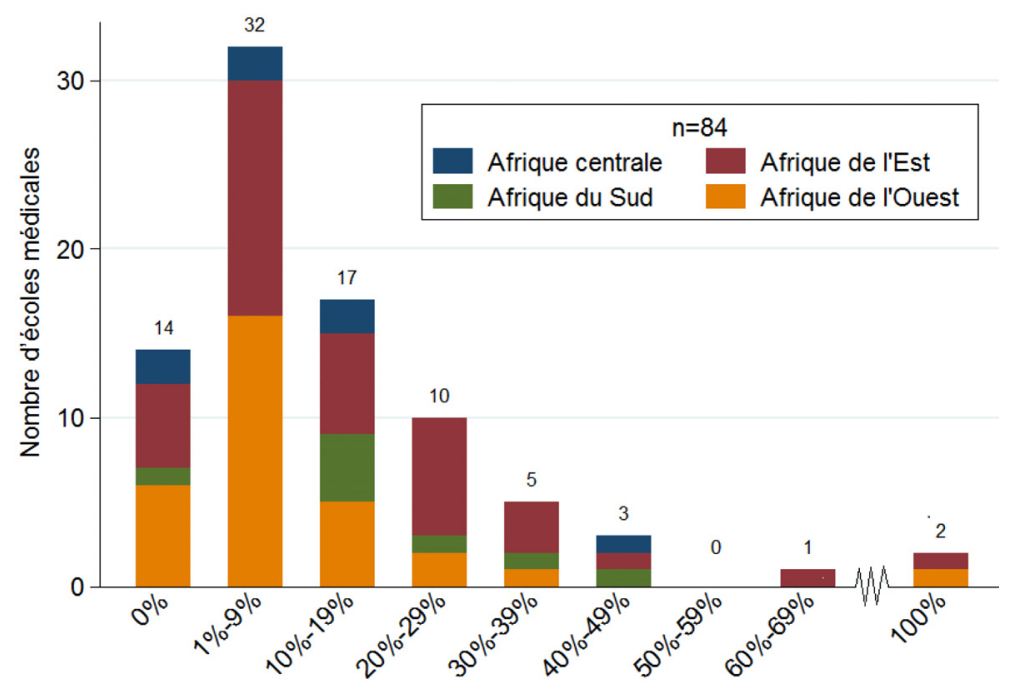

Fig. 6. Participation du corps professoral à la recherche financée par les subventions dans les écoles de médecine de l'Afrique subsaharienne, par région.

a introduit une formation en gestion pour les étudiants, reconnaissant que certains étudiants occuperont des postes administratifs en tant que responsables régionaux de santé publique ou médecin chefs d'hôpitaux une fois qu'ils seront diplômés.

\section{Importance de la recherche}

Au-delà de la création de nouvelles connaissances, la recherche est importante pour le développement des corps professoraux des écoles de médecine, la fidélisation du personnel et le renforcement de l'infrastructure. Nombre d'écoles ont déclaré que la recherche favorisait le recrutement et la fidélisation du personnel et attirait des partenaires extérieurs. Des écoles plus anciennes dotées de portefeuilles de recherche plus solides continuent à mettre au point des programmes de recherche grâce à des sources de financement existantes, permettant de former des membres plus jeunes du corps professoral. Pourtant, bien que les écoles bien établies réussissent à recevoir un appui financier pour la recherche, les écoles plus petites et récentes font face à des défis. Le personnel du corps professoral de ces écoles est souvent plus jeune et n'a reçu ni la formation ni l'encadrement nécessaires pour obtenir avec succès des subventions de recherches. En outre, les pénuries de personnel dans beaucoup d'écoles augmentent la charge de cours, limitant le temps disponible pour se livrer à la recherche. L'Université du Mali a volontairement programmé sa capacité de recherche sur 30 ans. Les premiers membres du corps professoral ont été envoyés à l'étranger pour une formation de troisième cycle et les diplômés qui revenaient étaient garantis d'avoir un soutien financier. Les membres actuels de l'équipe de recherche académique enseignent à l'école de médecine, ce qui est bénéfique à la fois pour les professeurs et pour les étudiants. L'University of Malawi récupère $10 \%$ des fonds indirects de tous les contrats de recherche afin de créer une base financière pour la recherche. Leur centre de soutien de la recherche assiste les professeurs pour la rédaction de demandes de subventions, la conception de la recherche et la gestion des subventions, ce qui, en retour, développe la capacité de recherche de l'université et augmente les salaires du corps professoral participant à recherche. Malgré ces exemples, dans la plupart des écoles de médecine en Afrique subsaharienne, moins de $10 \%$ des membres du corps professoral prennent part à une recherche subventionnée (figure 6). Nous avons comparé le pourcentage de membres participant à de la recherche financée par le biais de contrats 
avec une série de variables prédictives potentielles par régression linéaire multi variables. Bien que de nombreuses universités fournissent plusieurs types de soutien en matière de recherche (matériel éditorial complémentaire en ligne p. 15), seuls deux types (mise à disposition d'instruments de recherche institutionnels renforcés tels que des comités de recherche ou d'éthique ( $p=0,0002)$ et le financement du temps consacré à la recherche $(p=0,049))$ étaient nettement corrélés à une proportion accrue de membres du corps professoral participant à une recherche financée par des subventions. L'existence de programmes de formation à la recherche (qu'ils soient internes ou externes) n'était pas un facteur important. Nous avons remarqué des variations linguistiques révélant que les membres du corps professoral d'écoles utilisant l'anglais comme langue d'instruction avaient le plus de chances de participer à la recherche $(p=0,027)$ et que ceux d'écoles utilisant l'arabe avaient le moins de chances de le faire $(p=0,047)$.

\section{Innovations dans le curriculum}

Des innovations impressionnantes apparaissent dans les curriculums de nombre d'écoles. Les répondants à l'enquête ont fait état de plusieurs méthodes d'enseignement non traditionnelles utilisées dans les écoles de médecine en Afrique subsaharienne, parmi lesquelles une éducation axée sur la communauté, l'apprentissage par problèmes et l'apprentissage par équipes pluridisciplinaires (matériel éditorial complémentaire en ligne p. 16). Ces trois méthodes éducationnelles ont tendance à être mises en œuvre ensemble. Les institutions basant leurs programmes sur une part plus importante de l'une de ces méthodes d'instruction, quelle qu'elle soit, pendant les années précliniques avaient plus de probabilité d'utiliser les autres méthodes pendant cette période ( $p<$ 0,0001 pour chaque corrélation) et les institutions privilégiant l'apprentissage par équipes pluridisciplinaires lors des années cliniques avaient la plus forte probabilité d'utiliser davantage l'apprentissage par problèmes ( $p=0,0003)$ et axé sur la communauté $(p<0,0001)$ pendant cette période.

Une expérience structurée au sein de la communauté dans le cadre d'une éducation axée sur la communauté permet aux étudiants de se familiariser avec les populations mal desservies et prépare mieux les diplômés à appréhender les problèmes de santé nationaux. À la Jimma University, une perspective orientée vers la communauté est le pivot de la mission éducationnelle, qui débute par un programme de formation axé sur la communauté. Les étudiants en médecine commencent à travailler au sein de la communauté après avoir acquis la maîtrise de tâches incluant la collecte de données, le diagnostic communautaire, l'analyse et la préparation d'un plan d'intervention pour les problèmes locaux. À la faculté des sciences médicales de la Makerere University, en Ouganda, un nouveau curriculum inclut un contact régulier avec des patients des communautés rurales pendant toute la période des études de médecine.

À la Gezira University, le curriculum inclut une orientation communautaire, qui sert de principe organisateur pour les étudiants en médecine, le corps professoral et les diplômés. Un sens de responsabilité sociale accompagne tous les aspects de l'éducation, et la mission de l'école orientée sur la communauté est présentée de façon bien visible à l'entrée du bâtiment de l'école de médecine. Lors de la visite de l'équipe à l'université, les membres du corps professoral spécialisés en oncologie, néphrologie et pédiatrie ont fait état de l'effet continu des principes axés sur la communauté dans leur travail.

\section{Importance d'un programme de troisième cycle (post-gradué) en éducation médicale}

Le troisième cycle des études médecine est un aspect important de la stratégie de développement d'un système de santé national. Parmi les 96 répondants qui ont parlé de leurs programmes de troisième cycle, 58 ont dit qu'ils offraient des programmes cliniques post-gradués et 47 ont donné le nombre de 
diplômés qui sortaient de ces programmes par an. Ces 47 programmes correspondaient à 1909 places pour des programmes cliniques de troisième cycle, ce nombre de place ne permettant d'accueillir que moins de $25 \%$ du total des 7861 diplômés concernés par l'enquête. Les programmes de troisième cycle les plus couramment offerts étaient la médecine interne $(14 \%$ des places dites disponibles pour les études médicales de troisième cycle), l'obstétrique et la gynécologie (13\%), la pédiatrie (13\%), les sous-spécialités de chirurgie $(11 \%)$ et la chirurgie générale $(11 \%)$.

La Makerere University et l'Ibadan University offrent des systèmes de troisième cycle d'études de médecine bien développés. D'autres écoles ont moins de possibilités pour les étudiants de troisième cycle, et l'offre concerne alors souvent la pédiatrie, la chirurgie et l'obstétrique/gynécologie, tandis que quelques unes n'ont aucun de ces programmes. En augmentant le nombre de ces derniers, les écoles ont pu fidéliser davantage de diplômés et employer certains des diplômés récemment formés comme professeurs à la faculté. Certaines écoles mettent en œuvre ce que l'on appelle des programmes d'éducation de troisième cycle «sandwich » dans lesquels les internes poursuivent le programme sur place mais effectuent une partie de leur formation au sein d'un programme régional ou international. Ce principe vise à exposer les étudiants au travail clinique à l'étranger tout en atténuant la tendance des médecins à rester à l'étranger lorsque toute leur formation se déroule ailleurs.

\section{Qualité des écoles secondaires}

La variabilité de la qualité des écoles secondaires crée des problèmes pour les admissions aux écoles de médecine. Des systèmes solides d'éducation secondaire sont des conditions nécessaires pour assurer la réussite au sein des écoles de médecine mais dans nombre de pays la qualité de l'éducation secondaire n'est pas à la hauteur. C'est pourquoi certaines écoles ont mis en place des programmes préparatoires ou de recrutement pour les élèves défavorisés. La Catholic University, la Hubert Kairuki Memorial University et le College of Medicine au Malawi ont mis en œuvre des années préparatoires pour aider les élèves à être au niveau de l'enseignement des écoles de médecine. La Walter Sisulu University a établi des quotas par origine ethnique afin de refléter les caractéristiques socioéconomiques d'Afrique du Sud et évalue les candidats à l'aide d'un système de notes qui inclut la motivation et l'engagement au service. En outre, le curriculum du premier trimestre offre des cours de langue, une formation en informatique, des cours de rattrapage en sciences fondamentales et une attention particulière sur la façon d'étudier. Un programme de mentorat entre pairs aide les étudiants à s'adapter à la vie de campus.

\section{2. Écoles de médecine privées}

Malgré les défis à relever, les écoles de médecine privées font partie de l'innovation et de la croissance qui se dessinent au sein de l'offre en éducation médicale en Afrique subsaharienne. Les premières écoles de médecine privées ont ouvert dans les années 90 et les écoles privées constituent désormais $21 \%$ (22 sur 105) de toutes les écoles qui ont répondu à l'enquête (figure 2). La recherche dans les bases de données semble indiquer qu'un tiers (21 sur 63) des écoles n'ayant pas répondu sont privées, ce qui impliquerait que $26 \%$ (43 sur 168) des écoles de médecine actuellement ouvertes sont privées. Les partisans avancent que la privatisation de l'éducation médicale s'aligne sur les tendances mondiales au regard de l'éducation, alors que d'autres répliquent que ce mouvement est surtout commercial et inévitablement inéquitable.

Deux des dix écoles visitées étaient des institutions privées. La Hubert Kairuki Memorial University est une institution à but non lucratif fondée en 1997, qui a la réputation de produire des médecins diplômés très compétents. Cette université a conclu un partenariat avec les hôpitaux publics de district afin de procurer aux étudiants d'autres sites d'enseignement cliniques. La Catholic University est 
une école confessionnelle à but non lucratif, fondée en 1995. L'université est un modèle de collaboration réussie avec le gouvernement du Mozambique, l'Église Catholique et plusieurs organisations internationales.

\section{Partenariats internationaux}

Les partenariats internationaux sont des ressources importantes pour nombre d'écoles de médecine. Pratiquement toutes les écoles de médecine en Afrique subsaharienne, parmi lesquelles toutes les écoles visitées, œuvrent en collaboration localement et internationalement, principalement avec des institutions en Europe, en Amérique du Nord et en Afrique (matériel éditorial complémentaire en ligne p. 17). L'Université du Mali travaille en coopération avec le gouvernement français, qui l'a aidée dans le cadre de ses programmes de services de santé axés sur la communauté et pour la formation en matière de santé publique. Le Mali a également mis au point des partenariats de recherche avec des universités européennes, nord-américaines et d'autres universités africaines. La Makerere University collabore depuis longtemps ave des organisations académiques et à but non lucratif étrangères. Ces associations ont contribué au renforcement des capacités de recherche et des programmes de formation de l'école. L'University of Malawi illustre la collaboration SudSud, grâce à sa participation aux programmes de formation conjointe par le biais de la Southern Africa Human Capacity Development Coalition (Coalition pour le développement des capacités humaines en Afrique du Sud).

\section{Discussion}

Nous avons constaté une croissance remarquable de l'offre en éducation médicale dans la région au cours des deux dernières décennies. Elle avait d'ailleurs commencé longtemps avant que la communauté internationale ne s'intéresse à la pénurie grave des professionnels de santé dans la région. La décision prise par nombre de pays d'investir dans la construction de nouvelles écoles de médecine et d'agrandir celles qui existent, l'intérêt intense que manifestent les jeunes au regard des études de médecine et la création d'écoles de médecine privées sont des éléments qui reflètent ce mouvement. Les obstacles rencontrés lors de cette amplification, pourtant, restent non négligeables. En l'absence d'effectifs de professionnels de santé plus importants et stables, il y a peu de chance de mettre fin à l'épidémie du Sida ou de réaliser les «Objectifs du millénaire pour le développement en Afrique ». De nombreuses organisations internationales (incluant le Plan d'urgence présidentiel pour l'aide au Sida, l'Organisation mondiale de la santé, la Banque mondiale, le Fonds mondial, les agences d'aide bilatérale et les philanthropies) ont abouti à cette conclusion ${ }^{[9]}$. L'attention mondiale portée actuellement sur l'amplification de l'effectif des professionnels de santé crée une opportunité favorable à l'éducation médicale en Afrique subsaharienne.

Les effectifs ubiquitairement insuffisants des membres du corps professoral et le mauvais état général de l'infrastructure des campus sont les observations les plus notables révélées par cette étude. Ces conclusions ne surprennent personne parmi ceux qui s'intéressent à l'éducation médicale dans la région. Les professeurs en sciences fondamentales et les cliniciens sont peu nombreux partout, limitant sérieusement le développement d'une éducation de qualité. Les manques les plus graves se trouvent souvent dans les laboratoires, les bibliothèques, les salles de classe, les salles de conférence et les foyers d'étudiants. Les faiblesses des ressources en nouvelles technologies éducatives et en bande passante sont particulièrement problématiques, empêchant les étudiants d'aller au-delà des anciennes méthodes d'apprentissage au profit des développements modernes basé sur Internet. L'étude a constaté la régularité et l'échelle de ces problèmes, établissant un point de référence à partir duquel les efforts de développement devront être examinés.

La convergence de l'attention mondiale et les caractéristiques précises des obstacles auxquels se heurte l'expansion de l'éducation médicale créent 
un moment opportun. Les autorités nationales et les partenaires internationaux œuvrant avec les universités et les écoles de médecine ont la possibilité de créer de nouvelles stratégies collaboratives en matière d'investissement, mettant au point des engagements à long terme pour un financement conjoint grâce à des mécanismes tels que des fonds de dotation pour l'amélioration du corps professoral, des fonds spéciaux pour les nouveaux laboratoires et les besoins informatiques, ou des financement d'ensemble pour appuyer les partenariats Nord-Sud ou Sud-Sud entre les universités.

Un aspect non prévu de cette étude est l'opportunité qu'elle a procurée aux responsables africains de l'éducation médicale de se rencontrer et de travailler ensemble. Deux fois auparavant (dans les années 60 et 90) une Association des écoles de médecine africaine avait été lancée mais n'avait pas été maintenue ${ }^{[10]}$. La plupart des politiques en matière d'éducation médicale en Afrique sont centrées sur des programmes et des problèmes internes aux pays, avec peu d'occasions de discuter les problèmes et d'envisager les solutions communs à l'échelon interétats. Cette étude, qui a offert aux responsables de l'éducation l'opportunité d'aller ailleurs en Afrique pour comparer les programmes et les politiques, s'est révélée être très instructive et très stimulante. Une association des écoles africaines de médecine revitalisée serait en position de jouer un rôle important dans l'expansion de l'éducation médicale en Afrique subsaharienne, incluant la collecte de données pour les programmes existants, les rapports sur les bonnes pratiques, le lancement d'initiatives pour l'accréditation et la certification et la mise à jour des informations concernant les écoles médicales actuelles et celles qui se sont créées. Cette étude a également observé un changement notable au regard du rôle de l'éducation médicale de troisième cycle en Afrique subsaharienne. Les postes au sein des programmes de troisième cycle d'études médicales ne sont pas nombreux dans la plupart des pays d'Afrique. Nombre de diplômés africains cherchant une formation plus poussée partent l'acquérir en Europe et en Amérique du Nord et beaucoup ne reviennent pas. Afin de former plus de diplômés en spécialités de base et de mettre fin à l'émigration des jeunes diplômés, de nombreux pays démarrent et élargissent des programmes d'éducation médicale de troisième cycle. Cette tendance sera essentielle pour constituer des cadres durables de médecins de qualité dans tous les pays et pour améliorer les corps professoraux existants des écoles de médecine.

L'étude ne reprenait pas le modèle d'un travail précédent mais a réussi à créer une stratégie unique de collecte de données, susceptible d'être applicable à des études dans d'autres régions géographiques dans lesquelles les informations sur l'éducation des professionnels de santé ne sont pas bien développées. Les caractéristiques essentielles de la structure de l'étude étaient : 1) la revue de la littérature et la réalisation d'entretiens avec des informateurs clés afin de faire l'état des connaissances de base ; 2) la mise en place d'un comité consultatif d'éducateurs et de responsables des politiques de la région pour être en mesure de dégager une perspective, des conseils, d'analyser les observations et de formuler des conclusions ; 3 ) la collecte des données avec des instruments qualitatifs (visites structurées des sites) et quantitatifs (enquête, engageant chaque fois la participation essentielle du comité consultatif et 4) un engagement solidaire (comité consultatif et secrétariat) au moment de l'analyse, de la rédaction, des corrections et de la publication.

\section{Limites}

Cette étude comporte plusieurs limites. Les documents publiés sur l'éducation médicale en Afrique étaient surtout en anglais. Les articles écrits en français étaient moins nombreux, et rares étaient ceux en portugais et en arabe. Bien que des membres du comité consultatif aient apporté leur aide pour tenter de trouver davantage d'articles en d'autres langues que l'anglais, il en a été trouvé peu. La prépondérance des membres et du personnel du comité anglophone consultatif limitait aussi l'usage de sources d'informations dans d'autres langues que l'anglais. Parmi les dix écoles visitées, deux (au Mali et en Côte d'Ivoire) étaient francophones (une de moins 
que la parité de la population ne le justifierait), six étaient anglophones, une était lusophone et une était de langue arabe. La sous-représentation des pays francophones a persisté malgré plusieurs tentatives d'établir des contacts avec des écoles de la République démocratique du Congo, du Sénégal et du Cameroun. De même, les réponses de l'enquête indiquaient une sous-représentation de certaines régions, avec des taux de réponse inférieurs dans des pays tels que la République démocratique du Congo, l'Angola et le Soudan. Vingt-deux écoles n'ont pas été soumises à l'enquête parce qu'elles n'ont été découvertes ou identifiées qu'une fois l'étude achevée.

Certaines limites de l'enquête sont également liées à la nature subjective de plusieurs questions et au caractère incomplet des réponses à plusieurs questions, de façon ponctuelle pour certaines écoles ou de manière convergente pour des écoles d'un même pays. Les questions portant par exemple sur la proportion de revenu provenant de diverses sources, sur les raisons de perte de personnel ou d'émigration des diplômés et sur les choix de pratiques des diplômés étaient souvent des estimations données par les répondants plutôt que des réponses basées sur des données factuelles. Ni l'enquête ni les visites de sites n'évaluaient les compétences des diplômés à cause de l'absence de normes régionales établies au regard de la qualité de l'éducation.

Les questions n'ayant pas obtenu de réponse dans les questionnaires qui ont été renvoyés se sont avérées problématiques. Il était compréhensible que certaines questions aient été omises par des écoles précises, par exemple lorsqu'elles concernaient les diplômés alors que les écoles n'en avaient pas encore. Lorsque les questions restaient sans réponse de manière inexpliquée, des tentatives étaient faites pour contacter les répondants et leur demander de finir de remplir le questionnaire. Le nombre de réponses à chaque question digne d'intérêt est donné pour chaque conclusion. Dans certains cas, des réponses contradictoires portant sur les exigences nationales étaient formulées par des écoles du même pays. Par exemple, pour huit pays possédant plusieurs écoles ayant répondu, des réponses incohérentes concernant l'obligation de prestations de service de la part des diplômés ont été recueillies.

\section{Conclusions et recommandations}

L'étude a analysé le mode de fonctionnement des écoles de médecine pendant une période de recherche de deux ans. Les membres du comité consultatif ont trouvé que les perspectives qu'ils avaient mises à jour dans ce travail méritaient des recommandations collectives concernant les actions qui devraient être entreprises pour favoriser et améliorer l'éducation médicale et, en retour, la santé de la population en Afrique subsaharienne. Un ensemble de recommandations a été rédigé, distribué à tous les participants de l'étude, débattu, modifié, et adopté lors d'une réunion d'étude à Dar es Salam, Tanzanie, le 16 avril 2010. Le groupe de travail a fait part de ces recommandations. Nous espérons que le travail de l'étude sur les écoles de médecine en Afrique subsaharienne et ses recommandations serviront de catalyseur pour une innovation et un investissement plus poussés en éducation médicale en Afrique subsaharienne. Cette action, à son tour, devrait contribuer à produire un effectif plus robuste pour une amélioration de la santé en Afrique.

\section{Contributions des co-auteurs}

Tous les auteurs ont contribué à la recherche rapportée dans cet article. FM, SF, FO, SRG, EB et CC ont contribué au projet de l'article; FM, SF, CC, SRG, TW et HR ont participé à la rédaction de l'article ; EB, CC, FM, SF et TW ont participé à l'analyse des données.

\section{Conflits d'intérêt}

FO, PR et NKS ont reçu une subvention de la Fondation de Bill \& Melinda Gates. HA, SC, EB, CC, JC, SBC, SF, SRG, TH-M, EH, LJ, FM, HR et TW ont reçu un salaire provenant indirectement de fonds octroyés par la Fondation de Bill \& Melinda Gates. AH, EB, MJ-MID, DEEA, JF, JI, MJ, GLM, EOO$\mathrm{O}$, FO et NKS ont reçu des honoraires provenant 
indirectement de la Fondation de Bill \& Melinda Gates. EH et A-JN ont reçu des honoraires d'experts conseils provenant indirectement de la Fondation de Bill \& Melinda Gates. AH, HA, SBC, EB, CC, JC, SC, MJ-MID, DEEA, JF, SF, SRG, EH, JI, MJ, MM, GLM, FM, A-JN, HR, EOO-O, FO, PR et NKS ont bénéficié d'une prise en charge de leurs frais de déplacements au Symposium sur l'éducation médicale africaine aux fins de l'étude. EB, CC, JC, MJ-MID, TH-M, JI, MJ, LJ, MM, GLM, FM, A-JN, EOO-O, FO, PR et NKS ont bénéficié d'une prise en charge de leurs frais de déplacement pour la première réunion d'étude à Kampala, Ouganda, aux fins de l'étude. CC, JC, JI, MM, GIM, FM, AN, HR, EOO- O, FO, PR et and NKS ont bénéficié d'une prise en charge de leur frais de déplacement pour les réunions dans le cadre des visites sur place, aux fins de l'étude. AH, EB, MJ, A-JN et NKS ont reçu des honoraires pour leur participation à la révision des activités telles que les comités de surveillance des données, l'analyse statistique, les comités de validation des critères d'évaluation, etc. $\mathrm{AH}, \mathrm{EB}$ et A-JN ont été rémunérés pour écrire ou réviser le rapport. AH et $\mathrm{EB}$ ont reçu un soutien administratif. Les auteurs suivants déclarent qu'ils ont reçu d'autres subventions ou attendent d'autres subventions de la Fondation de Bill \& Melinda Gates : $\mathrm{CC}$ ira en déplacement à une réunion à Kampala, Ouganda, en novembre 2010; NKS est sous-traitant dans le cadre d'une subvention pour le jumelage de la Makerere University avec la Johns Hopkins University; et MJ-MID et NKS ont reçu des fonds pour organiser les visites de sites par l'équipe de l'étude dans leurs institutions. Tous les autres auteurs déclarent qu'ils n'ont aucun conflit d'intérêt.

Remerciements. Ce travail a reçu le soutien de la Fondation de Bill \& Melinda Gates.

\section{Références}

1. WHO. Working together for health; the World Health Report 2006. Geneva: World Health Organization, 2006.
2. Joint Learning Initiative. Human resources for health: overcoming the crisis. Cambridge: Harvard University Press, 2004.

3. Zuvekas A, Nolan L, Tumaylle C. Impact of community health workers on access, use of services and patient knowledge and behaviour. Washington: Bureau of Primary Health Care, US Public Health Services, 1998. [On line] Disponible sur : http://aspe.hhs. gov/pic/reports/hrsa/6355.pdf (accessed June 24, 2010).

4. Mullan F, Frehywot S. Non-physician clinicians in 47 subSaharan African countries. Lancet 2007;370:2158-63.

5. WHO. Task shifting global recommendations and guidelines. Geneva: World Health Organization, 2008. [On line] Disponible sur :

http://data. unaids.org/pub/Manual/2007/

ttr_taskshifting_en.pdf (accessed June 24, 2010).

6. WHO. Report on the WHO/PEPFAR planning meeting on scaling up nursing and medical education. Geneva: World Health Organization, 2009. [On line] Disponible sur : http://www.who.int/hrh/ resources/scaling-up_planning_report.pdf (accessed June 24, 2010).

7. WHO. World health statistics 2010. Geneva: World Health Organization, 2010. [On line] Disponible sur : http://www . who.int/whosis/whostat/EN_ WHS10_Full.pdf (accessed June 24, 2010).

8. Population Division of the Department of Economic and Social Affairs of the United Nations Secretariat. World population prospects: the 2008 revision. March, 2009. [On line] Disponible sur : http://esa.un.org/unpp (accessed June 1, 2010).

9. WHO and Global Health Workforce Alliance. Scaling up, saving lives: Task Force for Scaling Up Education and Training for Health Workers. Geneva: World Health Organization and Global Health Workforce Alliance, 2008. [On line ] Disponible sur : http://www. who. int/workforcealliance/documents/Global_ Health\%2OFINAL\%20REPORT.pdf (accessed June 10, 2009).

10. Bowers JZ. Association of African medical schools. J Med Educ 1962;37:220-21.

** Pour de plus amples détails sur l'Étude sur les écoles de médecine en Afrique subsaharienne, voir http:// SAMSS. org

Correspondance et offprints : Abdel Karim Koumaré. Faculté de Médecine de Pharmacie et d'Odonto Stomatologie, Université de Bamako, Bamako, Mali et Institut Africain de Formation en Pédagogie, Recherche et Evaluation en Sciences de la Santé (IAFPRESS), Quartier du Fleuve, Bamako, BP 05 Koulouba, Mali. Mailto : koumareak@hotmail.com 